\title{
The Pattern of Publications on Cerebral Arteriovenous Malformations and Cavernomas in the Middle East and North Africa
}

\begin{abstract}
Background: Cerebrovascular malformations are encountered frequently in clinical practice, but not much is known about the pattern of publication in the Middle East and North Africa (MENA) countries. We aim to evaluate the status and pattern of publications of cerebral arteriovenous malformations (AVM) and cavernomas in the MENA. Materials and Methods: PubMed database was searched for publications on cerebral AVM and cavernomas in the MENA between 2009 and 2019. Results: We found only 94 publications in the MENA region out of 31,333 publications pertaining to AVMs $(0.3 \%)$. The highest publishing country was Turkey, with $50(53.1 \%)$ studies. The case report was the study design used most by authors with $59(62.7 \%)$ studies. The majority of publications were by neurosurgeons with 42 of 94 (42.4\%) papers. European journals ranked first in the number of published articles with 42 of 94 (46.1\%) articles. Conclusions: We found a limited number of publications on cerebral AVMs and cavernomas by MENA countries in the past decade. Research support and national/regional registries are important factors to improve the academic output on AVMs and cavernomas in the MENA region.
\end{abstract}

Keywords: Arteriovenous malformations, cavernous malformations, Middle East, North Africa, pattern, publications

\section{Introduction}

Cerebrovascular malformations are a group of rare conditions that includes arteriovenous malformations (AVM) and cavernous malformations. ${ }^{[1,2]}$

Cavernous malformations (cavernomas) are the most common type of cerebral vascular abnormalities and account for $(10 \%-25 \%)$ of all vascular malformations. ${ }^{[3]}$ Cavernomas are an abnormal cluster of distended blood spaces that are characterized by multiple dilated "caverns" of blood vasculature. These spaces have a high tendency to leak since they lack the tight cell-to-cell junctions in addition to the weak supportive structures from smooth muscle and elastin surrounding them. ${ }^{[4]}$

The incidence of cavernomas is estimated to be $0.4 \%-0.8 \%$ in the general population without clear gender predilection. They occur in both adults and children, though the mean patient age is in the fourth decade. ${ }^{[3]}$ Seizures were the most common presenting symptom with $44.4 \%$, followed

This is an open access journal, and articles are distributed under the terms of the Creative Commons Attribution-NonCommercial-ShareAlike 4.0 License, which allows others to remix, tweak, and build upon the work non-commercially, as long as appropriate credit is given and the new creations are licensed under the identical terms.

For reprints contact: WKHLRPMedknow_reprints@wolterskluwer.com by headache $(30.6 \%)$ and neurological deficits $(30.6 \%)$. Most of cavernomas were found in cerebral hemispheres $(66.7 \%)$, spinal cord $(13.9 \%)$, brain stem $(11.1 \%)$, and cerebellum $(11.1 \%){ }^{[5]}$

AVMs are abnormal complex tangles of blood vessels which cause numerous shunts between the arterial and venous systems. They frequently occur in the brain and to a lesser degree in the spinal cord.

Cerebral AVMs present with intracranial hemorrhage $(38 \%-71 \%)$, followed by seizures $(18 \%-40 \%)$ and headaches $(5 \%-14 \%) .{ }^{[6]}$ AVMs occur in approximately $4 \%$ of the population and might be symptomatic in $12 \%$ of affected individuals. ${ }^{[7]}$ There is no gender predilection and are located supratentorially in $85 \%$ and infratentorially in $15 \%$ of patients.

The main options for the management of AVM include conservative management with serial imaging and observation, microsurgical resection, endovascular embolization, and stereotactic radiosurgery. ${ }^{[5]}$ The combined or staged

How to cite this article: Alolyani A, Alotaibi $\mathrm{H}$,
AlHamid MA, Alabbas F, Al-Jehani $\mathrm{H}$. The pattern of
publications on cerebral arteriovenous malformations
and cavernomas in the Middle East and North Africa.
Arab J Intervent Radiol 2020;4:92-5.

\section{Amira Alolyani, Horia Alotaibi, May Adel AlHamid', Faisal Alabbas ${ }^{2}$, Hosam Al-Jehani' ${ }^{2,3}$}

Department of Neurosurgery, College of Medicine, Imam Abdulrahman Bin Faisal University, Departments of ${ }^{l}$ Neurology and Interventional Radiology and ${ }^{2}$ Neurosurgery and Interventional Radiology, King Fahad Hospital of the University, Imam Abdulrahman Bin Faisal University, Dammam, Saudi Arabia, ${ }^{3}$ Department of Neurology and Neurosurgery, Montreal Neurological Institute and Hospital, McGill University, Montreal, Canada

Received: $13-04-2020$

Accepted: 08-06-2020

Online Published: 07-08-2020

Address for correspondence:

Dr. Hosam Al-Jehani,

C/O Luisa Birri, 3801

University St., Suite 109,

Montreal, QC, H3A 2B4,

Canada.

E-mail:hjehani@iau.edu.sa

Access this article online

Website: www.arabjir.com

DOI: 10.4103/AJIR.AJIR_11_20

Quick Response Code:

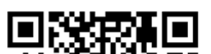

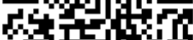

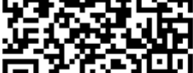

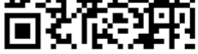


therapy is also gaining popularity through multidisciplinary decision-making approaches.

Given all these epidemiological and treatment option information presented in international literature, there is a considerable lack of such data in our Middle East and North Africa (MENA) region.

This study aims to evaluate the status of cerebral AVMs and cavernomas in MENA countries through the pattern and impact of their publications.

\section{Materials and Methods}

This study is based on a systematic review of the literature. PubMed database was comprehensively searched using the search strategy, as depicted in Table 1. Articles for this study were included if they were (1) written in English (2) published from January 1, 2009 to December 31, 2019 (3) related to cerebral AVMs and cavernomas, and (4) published in MENA countries by identification of the corresponding author's affiliations. We excluded letters to editors and studies that include vascular pathology other than cerebral AVMs and cavernomas. This was conducted by the first and senior author.

Several parameters were extracted from each article, such as title, year of publication, corresponding author's specialty and affiliation, study design, journal name, journal impact factor, and method of treatment. This was conducted by all the authors, the medical expertise of whom ranges from medical intern, to a neurovascular specialist to a neurovascular consultant.

\section{Results}

We found a total of 31,333 articles published in the English literature pertaining to AVMs. When we applied our MeSH criteria stated earlier, we retrieved a total of 274 published articles from the MENA region. After exclusions, only $94(0.3 \%)$ met our search criteria. The country with the highest number of publications was Turkey, with $50(53.1 \%)$ publications, followed by Israel $15(15.9 \%)$ then Iran $9(9.5 \%)$. The remainder of the countries is shown in Figure 1. No publications were found in Kuwait, Bahrain, Qatar, the United Arab Emirates, Oman, Yemen, Iraq, Syria, Jordan, Palestine, Algeria, Libya, or Sudan. Regarding publications by the type of study design, case reports were the most common study method used with $59(62.7 \%)$ studies [Figure 2]. When addressing the specialty of the main author, neurosurgeons ranked first with $42(42.4 \%)$ publications compared to the interventional radiologists with $29(29.2 \%)$ and other specialties with $28(28.2 \%)$ [Figure 3]. We found $42 \quad(46.1 \%)$ publications in European journals and 24 (26.3\%) in North American-based journals, followed by Middle Eastern and Asian journals, as shown in Figure 4. There has been a fairly steady distribution of the publication output between 2009 and 2019, with two peaks occurring in 2010 and 2017 with 14 and 16 publications, respectively [Figure 5].

Analyzing the modalities of treatment reported in the publication of the top three countries, we found that the majority of authors reported conservative management (45\%). Microsurgical resection was the second management strategy employed to treat these patients $(23 \%)$, followed by endovascular therapy (13\%) [Figure 6].

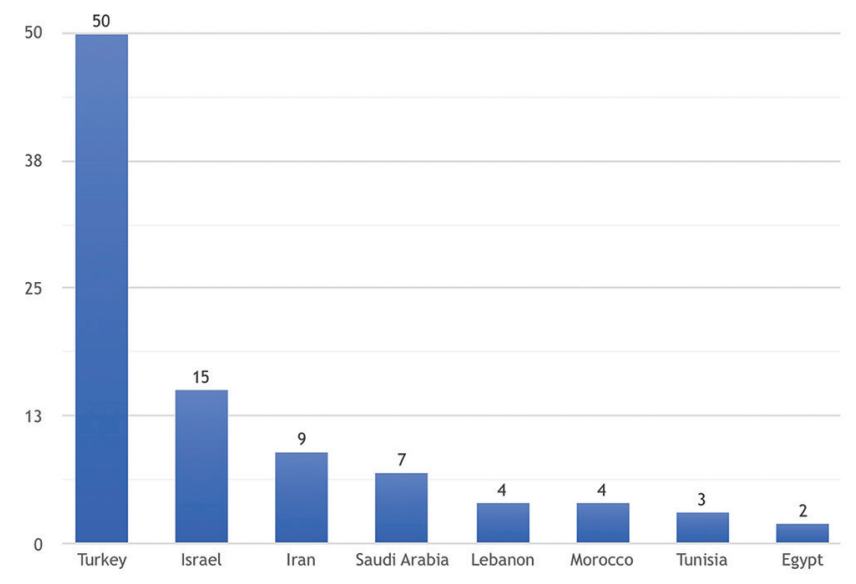

Figure 1: Publications by country

\begin{tabular}{|c|c|c|c|}
\hline Search terms & Best Match search information & $\begin{array}{c}\text { Number of } \\
\text { items obtained }\end{array}$ & $\begin{array}{c}\text { Number of items that } \\
\text { met the inclusion criteria }\end{array}$ \\
\hline $\begin{array}{l}\text { Cerebral cavernous malformation OR } \\
\text { intracranial cavernous malformation OR cerebral } \\
\text { cavernous hemangioma OR cerebral cavernoma } \\
\text { OR brain arteriovenous malformation OR } \\
\text { cerebral arteriovenous malformation OR } \\
\text { intracranial arteriovenous malformation) AND } \\
\text { (Saudi Arabia OR Kuwait OR Bahrain OR Qatar } \\
\text { OR United Arab Emirates OR Oman OR Yemen } \\
\text { OR Iraq OR Syria OR Lebanon OR Jordan OR } \\
\text { Israel OR Palestine OR Egypt OR Tunisia OR } \\
\text { Morocco OR Algeria OR Libya OR Sudan OR } \\
\text { Iran OR Turkey) }\end{array}$ & $\begin{array}{l}\text { MeSH Terms: Qatar; Kuwait; Turkeys; } \\
\text { Cerebrum; Brain; Israel; Hemangioma, } \\
\text { Cavernous; Hemangioma, Cavernous, } \\
\text { Central nervous system; Iraq; Egypt; } \\
\text { Syria; arteriovenous malformations; } \\
\text { Jordan; Algeria; Congenital abnormalities; } \\
\text { Iran; Saudi Arabia; Libya; the United } \\
\text { Arab Emirates; Lebanon; Yemen; } \\
\text { Morocco; Oman; Sudan; Tunisia; Turkey; } \\
\text { Intracranial arteriovenous malformations; } \\
\text { Bahrain } \\
\text { Subheading: Abnormalities }\end{array}$ & 274 & 94 \\
\hline
\end{tabular}




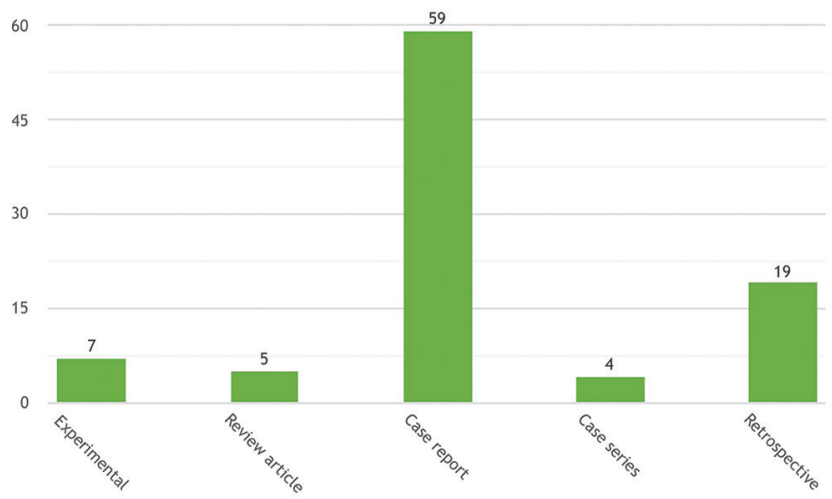

Figure 2: Publications by the type of study design

$$
50
$$

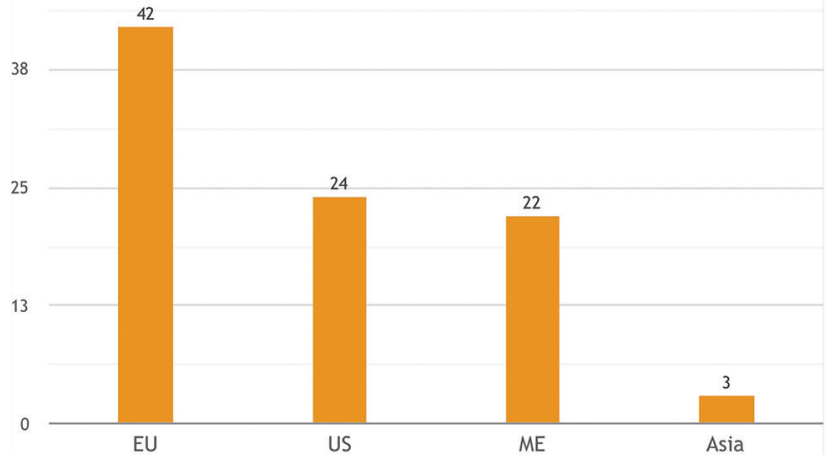

Figure 4: Publications by the region of journal

With regard to gross domestic product (GDP) and current health expenditure (CHE) and influence on publications, two of the top three countries, namely Turkey and Iran, were middle-class income countries with nearly equal CHE per capita of USD 444.7 and 475.5, respectively.

\section{Discussion}

Currently, improving the organization and the outcome of health-care services remains a crucial issue of concern in the MENA region. MENA region covers an area of around 11 million $\mathrm{km}^{2}$ and contains approximately $5.7 \%$ of the world's population. The epidemiological and demographic variations have a major influence on the organization and the outcome of health care in MENA countries. ${ }^{[8]}$ Health care in MENA countries is mostly paid by governments, spending about $58 \%$ of the national total health-care expenditures. The recent years have witnessed a rapid growth of government expenditure on health-care facilities in addition to improving quality, equity, and access. ${ }^{[9]}$

These and other issues contribute to the lack of publications in the MENA region. This has been reported in the interventional literature in recent years. ${ }^{[10]}$

Based on our results, there were only 94 published articles on cerebral AVMs and cavernomas by MENA in the past

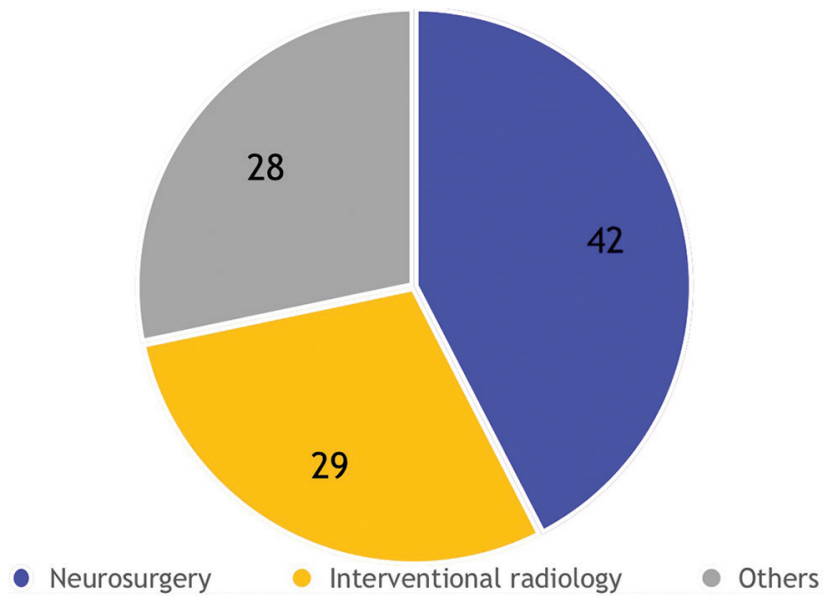

Figure 3: Publications by the main author specialty

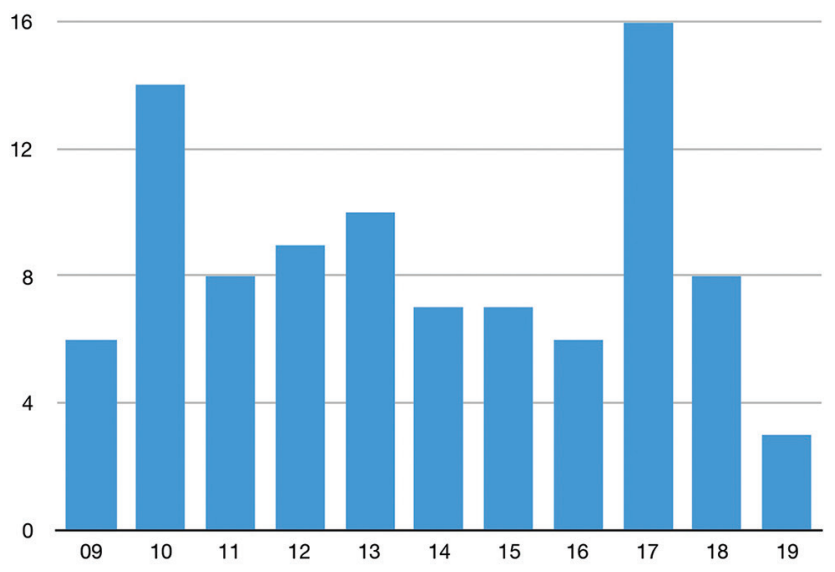

Figure 5: Distribution of publication by year

10 years out of 31.33 publications worldwide. This represents a small percentage of the bibliographic impact of the MENA region in the AVM domain. The highest publishing country was Turkey (50 [53.1\%]) followed by Israel (15 [15.9\%]) then Iran (9 [9.5\%]) [Figure 1]. This low number of publications can be attributed to several factors, including the limited number of physicians interested in academic publishing, poor research support, and limited disease registries. ${ }^{[1]}$ There are strict institutional rules and regulations that might limit the collaboration between different health-care sectors. This might, at least in theory, limit the access to data needed for future high-caliber publications.

When addressing publications by the region of the journal, we found that the majority of authors chose European journals over local journals ( $46.1 \%$ vs. $23.4 \%)$. This could be related to physicians' preference to publish in journals with a higher impact factor [Figure 4].

When analyzing the articles by the specialty of the corresponding author, it was found that neurosurgeons published most of the studies (42 [42.4\%]) compared to interventional radiologists (29 [29.2\%]) [Figure 3], which 


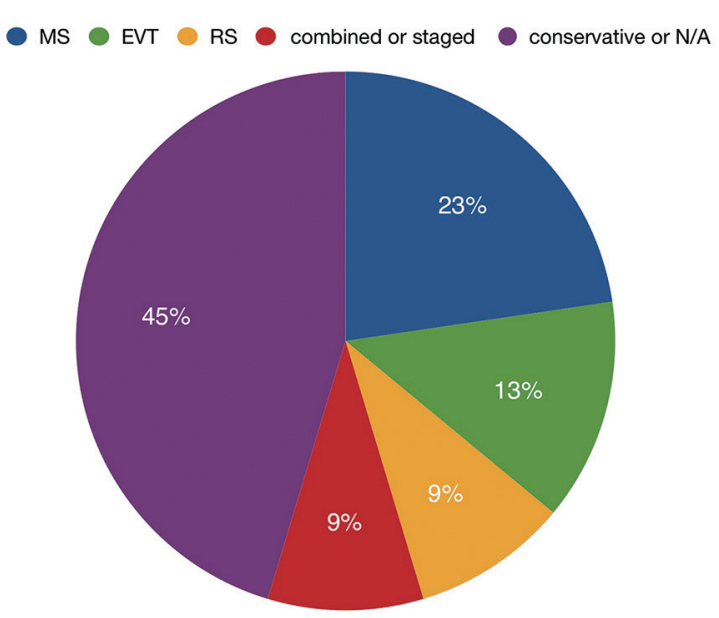

Figure 6: Distribution of modality of management in the 3 top publishing countries

could be explained, in part, by the current structure of practice in the MENA countries where the interventional radiologists are not the primary physicians for these patients. ${ }^{[12]}$

Regarding publications by the type of study design, the case report was found to be the most common study method used with $59(62.7 \%)$ articles, followed by retrospective study (19 [20.2\%]) and experimental study (7 [7.4\%]) respectively [Figure 2]. This is a reflection on the lack of a structured environment to conduct high-impact research projects in the MENA region.

There was no correlation between GDP and CHE per capita and the number of publications in each country, as two of the top three countries were middle-class income countries with nearly equal CHE per capita among them. This further consolidates the notion that most of these publications are driven by individual physicians' efforts rather than a structured national process.

The limitation of our study is that we limited our search to PubMed/Medline database. This strict criterion was applied to avoid reporting low-quality publications.

Despite this limitation, we could glean noteworthy findings, based on which we can propose that the role of national databases should be increased. This will allow for better evaluation systems for researches and researchers, more funding options, and more productive academic institution output.

There is a clear need to expand on AVM publications in the MENA region, which would provide a better reflection of the current regional health-care practices. To that end, we emphasize the need to establish comprehensive national or regional teams or multidisciplinary practice groups that follow the AVM patients collaboratively to improve upon treatment outcomes. In addition, there is a pressing need for construction and maintenance of disease registries, which would be of great benefit for better data collection and tracking of quality indicators for health-care delivery in the MENA region.

\section{Conclusion}

We found a limited number of publications on cerebral AVMs and cavernomas by MENA countries in the past decade. Research support and national/regional registries are important factors to improve the academic output on AVMs and cavernomas in the MENA region.

\section{Acknowledgments}

The authors wish to thank the College of Medicine, Imam Abdulrahman Bin Faisal, King Fahad Hospital University, for supporting this research

Financial support and sponsorship

Nil.

\section{Conflicts of interest}

There are no conflicts of interest.

\section{References}

1. Arteriovenous Malformations and Other Vascular Lesions of the Central Nervous System Fact Sheet. National Institute of Neurological Disorders and Stroke; 2019. Available from: https:// www.ninds.nih.gov/Disorders/Patient-Caregiver-Education/FactSheets/Arteriovenous-Malformation-Fact-Sheet. [Last updated on 2020 Jun 29].

2. Joshua A, Bartlett E, Edward I. Vascular malformations: A review. Semin Plast Surg 2014;28:58-63.

3. Zyck S, Gould GC. Cavernous Venous Malformation. In: StatPearls. Treasure Island (FL): StatPearls Publishing; 2019. Available from: https://www.ncbi.nlm.nih.gov/books/ NBK526009/. [Last updated on 2019 Dec 09].

4. Akers A. Cavernous Malformation. National Organization for Rare Disease; 2016. Available from: https://rarediseases.org/ rare-diseases/cavernous-malformation/\#supporting-organizations. [Last updated on 2020 Jun 29].

5. Karri SB, Uppin MS, Rajesh A, Ashish K, Bhattacharjee S, Jyotsna Rani Y, et al. Vascular malformations of central nervous system: A series from tertiary care hospital in South India. J Neurosci Rural Pract 2016;7:262-8.

6. Ajiboye N, Chalouhi N, Starke RM, Zanaty M, Bell R. Cerebral arteriovenous malformations: Evaluation and management. ScientificWorldJournal 2014;2014:649036.

7. Festa JR. Neurovascular Neuropsychology. New York: Springer Verlag; 2009.

8. Hamidi S, Akinci F. Measuring efficiency of health systems of the Middle East and North Africa (MENA) region using stochastic frontier analysis. Appl Health Econ Health Policy 2016;14:337-47.

9. Dhaoui I. Healthcare system efficiency and its determinants: A two-stage Data Envelopment Analysis (DEA) from MENA countries. Econ Res Forum 2019;1320:48.

10. Al Zahrani Y, Arabi M. Arab's research productivity and contribution to vascular and interventional radiology literature. Arab J Intervent Radiol 2017;1:27-9.

11. Akala FA, El-Saharty S. Public-health challenges in the Middle East and North Africa. Lancet 2006;367:961-4.

12. Al-Kutoubi A. Admission privileges and clinical responsibilities for interventional radiologists. Cardiovasc Intervent Radiol 2015;38:257-60. 